regression is significant $(\mathrm{P}=<\mathrm{O} \cdot \mathrm{OI})$ and has a slope not significantly different from I $\cdot 0$. Only the removal of $S$. vavilovii from the $6 \times 6$ data gives this result and it is concluded therefore that the interaction, in the main at least, is between $S$. vavilovii and the other species, whose genes act in additive fashion with dominance and little or no interaction. In terms of dominance, $S$. ancestrale has most dominant genes and in decreasing order, S. dighoricum, $S$. montanum, $S$. cereale, and $S$. turkestanicum.

For this character the analysis provides therefore a classification of species differences in purely genetical as distinct from morphological or physiological terms. Similar analyses have been carried out, and are being carried out, on a number of other morphological and cytological characters in these species. These analyses help towards gaining a better understanding of the nature of specific differences and provide, wherever practicable, an extremely useful device that could be widely used for such investigations.

\title{
REFERENCE
}

JINKS, J. L. 1954. The analysis of continuous variation in a diallel cross of Nicotiana rustica varieties. Genetics, 39, 767-788.

\section{AN EXTENSION OF THE USE OF ORTHOGONAL COEFFICIENTS IN PARTITIONING GENETIC VARIANCES}

\author{
M. DALEBROUX \\ European Community of Atomic Energy, Department of Biology, \\ Institute of Genetics, University of Pavia, Italy
}

\section{INTRODUCTION}

Received 15.v.62

In this paper, we propose to develop a method for partitioning genetic variances in diploids and autotetraploids with two alleles. $\mathrm{Li}$ (1957) has reported a method for partitioning the variances of such genotypic arrays, which he called "the method of successive linear regressions". This method can of course be extended to higher auto-2n-ploids with two alleles.

\section{THE USE OF ORTHOGONAL COEFFICIENTS}

We now consider a different approach to partitioning the genetic variance, which makes use of orthogonal coefficients. Cockerham (1954) presents the partitioning of the variance of a two-factor genotypic array in diploids when epistasis is present. In order to work out the breakdown of the total variance, he makes use of orthogonal coefficients. By subtracting the linear component from the total genetic variance of a diploid array, we get the quadratic component which cannot be anything but the dominance component; thus, if we break down the variance of a diploid genotypic array into its linear and quadratic components by means of a suitable set of orthogonal coefficients, then it is clear that we must get genetically meaningful components, i.e. the additive and dominance components.

We shall show that the method remains valid for an autotetraploid array with two alleles, i.e., we shall prove that a suitable orthogonal partitioning of the variance leads, in this latter case, to the conclusion that the two-, three- and four-gene effects are in fact regressions of the second, third and fourth degree respectively. 
We first calculate the orthogonal coefficients (see Grandage, I95 8 and $\mathrm{Li}$, 1959) which are necessary for determining the first degree response in an autotetraploid array with two alleles. These are found to be :

$$
\xi_{1 i}:-4 p, q-3 p, \quad 2(q-p), \quad 3 q-p \text { and } 4 q \text {, }
$$

where $p$ and $q$ are the relative frequencies of $\mathrm{A}$ and a respectively.

Let us weight the genotypic values by their respective frequencies. The aaaa, Aaaa, AAaa, AAAa and AAAA genotypes are coded as o, I, 2, 3 and 4 respectively, while their genotypic values are $Y_{0}, Y_{1}, Y_{2}, Y_{3}$ and $Y_{4}$ respectively. Table I summarises these relationships.

TABLE 1

\begin{tabular}{|c|c|c|c|c|}
\hline Genotypes & Code & $\begin{array}{c}\text { Relative frequencies } \\
f_{i}\end{array}$ & $\begin{array}{c}\text { Genotypic values } \\
Y_{i}\end{array}$ & $\begin{array}{c}\text { Weighted genotypic } \\
\text { values : } y_{i}\end{array}$ \\
\hline & & $q^{4}$ & $Y_{0}$ & $q^{4} Y_{0}$ \\
aaaa & 0 & $4 p q^{3}$ & $Y_{1}$ & $4 p q^{3} Y_{1}$ \\
Aaaa & 1 & $6 p^{2} q^{2}$ & $Y_{2}$ & $6 p^{2} q^{2} Y_{2}$ \\
AAaa & 2 & $4 p^{3} q$ & $Y_{3}$ & $4 p^{3} q Y_{3}$ \\
AAAA & 3 & $p^{4}$ & $Y_{4}^{4}$ & \\
\hline
\end{tabular}

Now,

$$
\begin{aligned}
& \mathrm{S} y_{i} \xi_{1 i}= 4 p q\left[p^{3}\left(\mathrm{Y}_{4}-\mathrm{Y}_{3}\right)+3 p^{2} q\left(\mathrm{Y}_{3}-\mathrm{Y}_{2}\right)+3 p q^{2}\left(\mathrm{Y}_{2}-\mathrm{Y}_{1}\right)+q^{3}\left(\mathrm{Y}_{1}-\mathrm{Y}_{0}\right)\right] \\
&=4 p q \alpha, \text { since the expression in brackets is the } \alpha \text { value found by } \\
& \text { Li's method }(1957) \text { to work out the calculation of the } \\
& \text { linear component of the variance in an autotetraploid } \\
& \text { with two alleles. }
\end{aligned}
$$

and

$$
\sigma_{\mathrm{L}}^{2}=\frac{\left(\mathrm{S} y_{i} \xi_{1 i}\right)^{2}}{\mathrm{~S} f_{i} \xi_{1 i}^{2}}=\frac{(4 p q \alpha)^{2}}{4 p q}=4 p q \alpha^{2}
$$

In a similar way, it can be shown that :

$\sigma_{\mathrm{D}}^{2}=6 p^{2} q^{2} \beta^{2}$ two-gene effect or quadratic component,

$\sigma_{T}^{2}=4 p^{3} q^{3} \gamma^{2}$ three-gene effect or cubic component

and $\sigma_{\mathrm{F}}^{2}=p^{4} q^{4} \delta^{2}$ four-gene effect or quartic component,

where $\beta, \gamma$ and $\delta$ are the values found by Li's method (I957) to calculate the two-, three- and four-gene effects.

We see that the method of orthogonal coefficients, as presented here, leads to the correct partitioning of the genetic variance into its single components. We may therefore conclude that, in autotetraploid systems with two alleles, the two-, three- and four-gene effects are really regressions of the second, third and fourth degree respectively.

It is clear that this type of orthogonal analysis is likely to lead to a similar breakdown of the genetic variance in any auto-2n-ploid system with two alleles.

\section{TWO-FACTOR EPISTASIS WITHOUT LINKAGE}

Since it is proved that the partitioning of the genetic variance in autotetraploids with two alleles may be worked out by means of orthogonal coefficients, we can, by definition, calculate the epistasis effects in the same way. 
NOTES AND COMMENTS

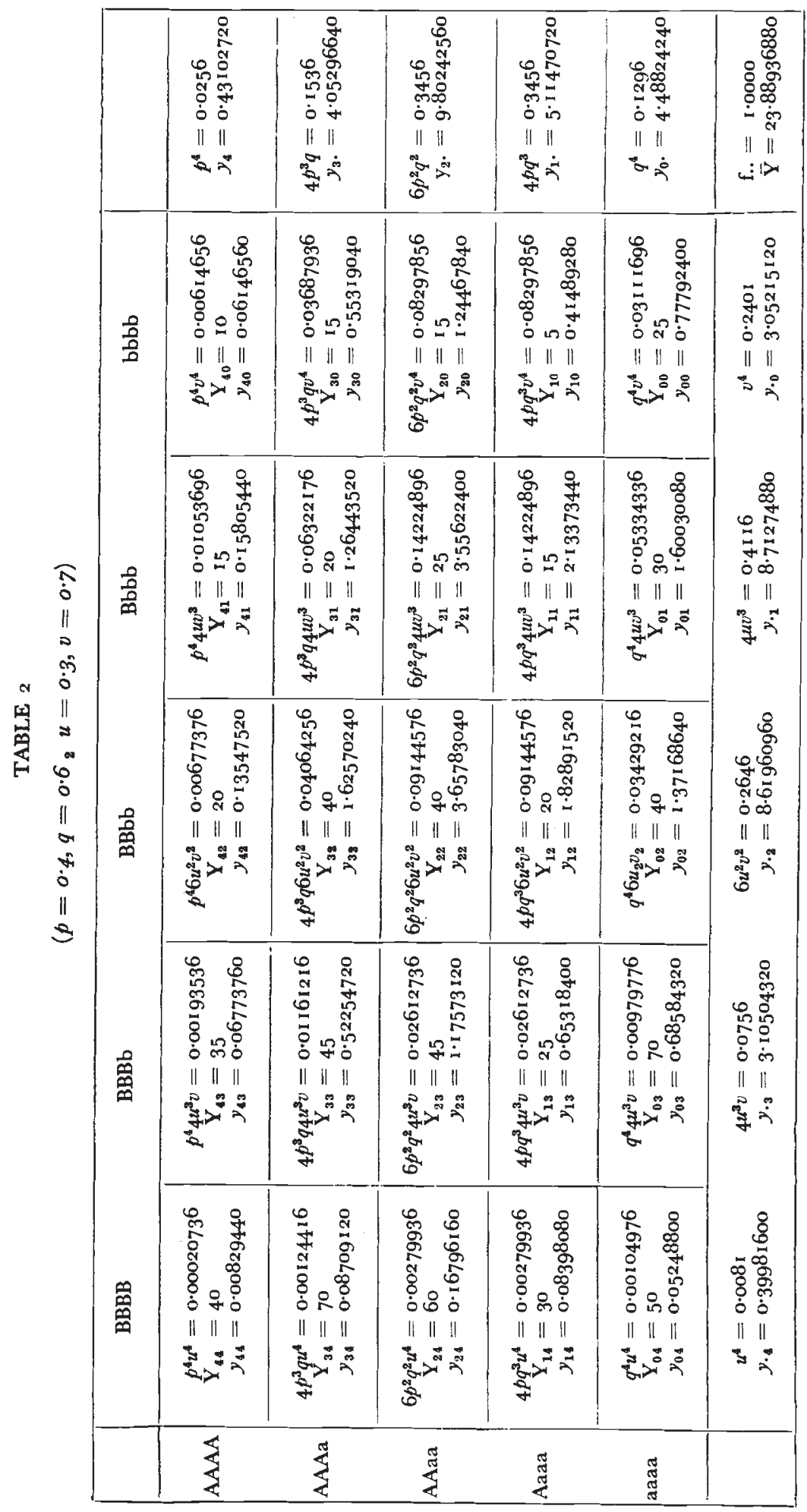


We shall calculate a numerical example based on the data listed in table 2.

The example is concerned with a two-locus epistasis, assuming that the frequencies at one locus are not correlated with those at the other. Table 2 shows the data required for the calculation.

According to the marginal frequencies of table 2, the orthogonal coefficients needed are given in table 3 .

TABLE 3

\begin{tabular}{|c|c|c|c|c|c|c|}
\hline Genotypes & Code & $f_{i}$ & $\xi_{1 i}$ & $\xi_{2 i}$ & $\xi_{1 i}$ & $\xi_{4 i}$ \\
\hline aaaa & 0 & O. I 296 & -8 & 24 & -32 & I 6 \\
\hline Aaaa & I & 0.3456 & -3 & -6 & 28 & -24 \\
\hline AAaa & 2 & 0.3456 & 2 & $-I I$ & -12 & 36 \\
\hline $\begin{array}{l}\text { AAAa } \\
\text { AAAA }\end{array}$ & $\begin{array}{l}3 \\
4\end{array}$ & $\begin{array}{l}0.1536 \\
0.0256\end{array}$ & $\begin{array}{r}7 \\
12\end{array}$ & $\begin{array}{r}9 \\
54\end{array}$ & $\begin{array}{r}-27 \\
108\end{array}$ & $\begin{array}{r}5 \\
-54 \\
81\end{array}$ \\
\hline bbbb & o & 0.2401 & -6 & 27 & -54 & $8 I$ \\
\hline $\mathrm{Bbbb}$ & I & 0.4116 & -1 & -18 & 81 & -189 \\
\hline $\mathrm{BBbb}$ & 2 & 0.2646 & 4 & -13 & -84 & 441 \\
\hline $\mathrm{BBBb}$ & 3 & 0.0756 & 9 & $4^{2}$ & -49 & -1029 \\
\hline BBBB & 4 & $0.008 \mathrm{I}$ & 14 & 147 & 686 & 2401 \\
\hline
\end{tabular}

The total genetic variance of the array represented in table 2 is :

$$
\sigma_{\mathrm{Y}}^{2}=\mathrm{S} f_{i} \mathrm{Y}_{i}^{2}-\overline{\mathrm{Y}}^{2}=\mathrm{I} 45 \cdot 262
$$

This variance is partitioned into its 24 single components, which finally reduce to the 14 following ones :

Main effects

$\left.\begin{array}{l}\sigma_{\mathrm{L}}^{2} \text { additive component } \\ \sigma_{\mathrm{D}}^{2} \text { two-gene effect } \\ \sigma_{\mathrm{T}}^{2} \text { three-gene effect } \\ \sigma_{\mathrm{F}}^{2} \text { four-gene effect }\end{array}\right]$ dominance effects $\left[\begin{array}{r}80 \cdot 180 \\ 3 \cdot 892 \\ 38 \cdot 286 \\ 10 \cdot 886\end{array}\right.$

Epistasis effects

$$
\begin{array}{ll}
\sigma_{\mathrm{LL}}^{2}=0.687 & \sigma_{\mathrm{LD}}^{2}=0.068 \\
\sigma_{\mathrm{LT}}^{2}=3.609 & \sigma_{\mathrm{LF}}^{2}=2.912 \\
\sigma_{\mathrm{DD}}^{2}=1.638 & \sigma_{\mathrm{DT}}^{2}=0.963 \\
\sigma_{\mathrm{DF}}^{2}=1.040 & \sigma_{\mathrm{TT}}^{2}=0.869 \\
\sigma_{\mathrm{TF}}^{2}=0.093 & \sigma_{\mathrm{FF}}^{2}=0.136
\end{array}
$$

Total $=145 \cdot 259=$ total genetic variance which is not exactly $145 \cdot 262$ because of the rounding errors. If the calculation is worked out with all the decimals, i.e. 14, then the fourteen components add up to $145^{\cdot 262}$.

Any n-loci epistasis in autotetraploids and higher auto-2n-ploids with two alleles could be treated in the same way. 


\section{SUMMARY}

Cockerham (1954) develops a method, which is based on the concept of orthogonal coefficients, for partitioning hereditary variance of a diploid array with n-locus epistasis.

The author shows that the two-, three- and four-gene effects of the variance in an autotetraploid with two alleles are, in fact, regressions of second, third and fourth degree respectively. The total genetic variance in any auto-2n-ploid with two alleles is likely to be broken down into genetically meaningful components by the method of orthogonal coefficients, which partitions the total variance into its first, second, $\ldots, 2 n$th degree regression components.

A numerical example of partitioning the variance of an autotetraploid (with two alleles) array with two-loci epistasis is worked out. Any n-loci epistasis in autotetraploids and higher auto-2n-ploids with two alleles could be treated in the same way.

Acknowledgments. - We are most grateful to Professor K. Mather, of the University of Birmingham, for having read the manuscript. We also thank Dr A. W. F. Edwards, of the University of Pavia, for having corrected the English of the manuscript.

\section{REFERENCES}

COCKERHAM, C. C. 1954. An extension of the concept of partitioning hereditary variance for analysis of covariances among relatives when epistasis is present. Genetics, 39, 859-882.

GRANDAGE, A. 1958. Orthogonal coefficients for unequal intervals. Biometrics, I4, 287-289.

LI, c. c. 1957. The genetic variance of autotetraploids with two alleles. Genetics, 42, 583-592.

LI, c. C. 1959. Numbers from experiments; A basic analysis of variation. The Boxwood Press, Pittsburgh 13, Pennsylvania. 